\title{
In-gas laser ionization and spectroscopy of actinium isotopes near the $N=126$ closed shell
}

C. Granados, P. Creemers, R. Ferrer, L. P. Gaffney, W. Gins, R. de Groote, M. Huyse, Yu. Kudryavtsev, Y. Martínez, S. Raeder, S. Sels, C. Van Beveren, P. Van den Bergh, P. Van Duppen, K. Wrzosek-Lipska, and A. Zadvornaya KU Leuven, Instituut voor Kern- en Stralingsfysica, B-3001 Leuven, Belgium

\section{A. E. Barzakh}

Petersburg Nuclear Physics Institute, NRC Kurchatov Institute, 188300 Gatchina, Russia

B. Bastin, P. Delahaye, L. Hijazi, N. Lecesne, F. Luton, J. Piot, H. Savajols, J.-C. Thomas, and E. Traykov GANIL, CEA/DSM-CNRS/IN2P3, B.P. 55027, 14076 Caen, France

R. Beerwerth and S. Fritzsche

Helmholtz Institute Jena, Fröbelstieg 3, 07743 Jena, Germany and Theoretisch-Physikalisches Institut, Friedrich Schiller Universität Jena, D-07743 Jena, Germany

M. Block

GSI Helmholtzzentrum für Schwerionenforschung GmbH, 64291 Darmstadt, Germany; Helmholtz-Institut Mainz, 55128 Mainz, Germany;

and Institut für Kernchemie, Johannes Gutenberg-Universität Mainz, Fritz-Straßmann-Weg 2, 55128 Mainz, Germany

X. Fléchard

Normandie Univ, ENSICAEN, UNICAEN, CNRS/IN2P3, LPC Caen, 14000 Caen, France

S. Franchoo

Institute de Physique Nucléaire (IPN) d'Orsay, 91406 Orsay, Cedex, France

L. Ghys

SCK-CEN, Belgian Nuclear Research Centre, Boeretang 200, 2400 Mol, Belgium and KU Leuven, Instituut voor Kern- en Stralingsfysica, B-3001 Leuven, Belgium

\section{H. Grawe}

GSI Helmholtzzentrum für Schwerionenforschung GmbH, 64291 Darmstadt, Germany

R. Heinke, T. Kron, P. Naubereit, and K. Wendt

Institut für Physik, Johannes-Gutenberg Universität, 55128 Mainz, Germany

M. Laatiaoui

GSI Helmholtzzentrum für Schwerionenforschung GmbH, 64291 Darmstadt, Germany and Helmholtz-Institut Mainz, 55128 Mainz, Germany

I. Moore and V. Sonnenschein

Department of Physics, University of Jyväskylä, P.O. Box 35 (YFL), Jyväskylä, FI-40014, Finland

M. Loiselet

Université Catholique de Louvain, Centre de Ressources du Cyclotron, B-1348 Louvain-la-Neuve, Belgium

$$
\text { E. Mogilevskiy }
$$

KU Leuven, Instituut voor Kern- en Stralingsfysica, B-3001 Leuven, Belgium

and Faculty of Mechanics and Mathematics, Lomonosov Moscow State University, Leninskie gory, 1, 119992, Moscow, Russia

S. Rothe

CERN, CH-1211 Genève, Switzerland

(Received 10 March 2017; revised manuscript received 13 July 2017; published 29 November 2017)

Published by the American Physical Society under the terms of the Creative Commons Attribution 4.0 International license. Further distribution of this work must maintain attribution to the author(s) and the published article's title, journal citation, and DOI. 


\begin{abstract}
The in-gas laser ionization and spectroscopy (IGLIS) technique was applied on the ${ }^{212-215} \mathrm{Ac}$ isotopes, produced at the Leuven Isotope Separator On-Line (LISOL) facility by using the in-gas-cell and the in-gas-jet methods. The first application under on-line conditions of the in-gas-jet laser spectroscopy method showed a superior performance in terms of selectivity, spectral resolution, and efficiency in comparison with the in-gas-cell method. Following the analysis of both experiments, the magnetic-dipole moments for the ${ }^{212-215}$ Ac isotopes, electricquadrupole moments and nuclear spins for the ${ }^{214,215} \mathrm{Ac}$ isotopes are presented and discussed. A good agreement is obtained with large-scale nuclear shell-model calculations by using a ${ }^{208} \mathrm{~Pb}$ core.
\end{abstract}

DOI: 10.1103/PhysRevC.96.054331

\section{INTRODUCTION}

Heavy nuclei, close to the doubly magic nucleus ${ }^{208} \mathrm{~Pb}$, are well described by large-scale nuclear-shell-model calculations indicating the robustness of the $Z=82$ and $N=126$ closed proton and neutron shells, respectively [1]. However, it is not clear when this description breaks down moving away from ${ }^{208} \mathrm{~Pb}$. While information on semimagic nuclei below ${ }^{208} \mathrm{~Pb}(\mathrm{Z}<82)$ or after the neutron closed shell $(\mathrm{N}>126)$ is limited, neutron-deficient lead isotopes have been studied in detail almost down to the proton drip line. The latter region of the nuclear chart is notorious for the manifestation of shape coexistence in atomic nuclei [2]. Information on the $N=126$ isotones above ${ }^{208} \mathrm{~Pb}$ is also limited, but recent work suggests a significant weakening of the $N=126$ shell closure in uranium $(Z=92)[3]$.

Magnetic-dipole moments $\mu$ and electrical quadrupole moments $Q$ are sensitive probes for the study of the singleparticle structure and collective behavior of nuclei and can be deduced from laser spectroscopy studies [4]. Investigations of those properties in heavy nuclei are, however, hampered by low production rates and short half-lives of the isotopes of interest. The in-gas laser ionization and spectroscopy (IGLIS) technique was applied at the Leuven Isotope Separator On-Line (LISOL) facility, to produce and investigate radioactive ion beams of high purity. In-gas-cell laser spectroscopy of neutrondeficient copper and silver isotopes has been performed with an average spectral resolution of 5 and $9 \mathrm{GHz}$, respectively [5,6]. Recently, the production and subsequent laser spectroscopy investigation of nobelium in a gas-cell system was also demonstrated with a similar spectral resolution [7]. However, because of this limited spectral resolution the full hyperfine structure is often unresolved, preventing the determination of basic nuclear ground-state properties such as spins and quadrupole moments. To overcome these limitations, the in-gas-jet laser spectroscopy method was proposed [8]. In this method, the spectral line broadening is reduced due to a supersonic expansion of a gas seeded with the element under investigation after extraction through a de Laval nozzle. The density and temperature reductions in the supersonic jet substantially decreases the collisional and Doppler broadening of the spectral lines to a few hundreds of MHz. In addition, the enhanced total efficiency under optimized experimental conditions enables the investigation of isotopes produced at very low production rates.

While information on the decay properties of the neutrondeficient ${ }^{212-215}$ Ac isotopes and, in some cases, on excited nuclear states is available [9], data on nuclear moments in the whole isotopic chain of actinium is only available for shortlived isomers in ${ }^{215} \mathrm{Ac}$ [10], the ground-state and high-spin isomer in ${ }^{217} \mathrm{Ac}$ [11] and the long-lived ${ }^{227} \mathrm{Ac}$ [12]. The first optical experiments on actinium isotopes were carried out on ${ }^{227}$ Ac more than sixty years ago, resulting in the identification of a series of atomic levels for actinium in neutral and ionic form. Assignments of the atomic energy levels, spectral terms, and the electronic configuration was possible [12] and the nuclear spin was deduced from the hyperfine structure (HFS) obtained in the same optical spectroscopy experiments [13]. The magnetic-dipole and electric-quadrupole moment of the ground-state of ${ }^{227} \mathrm{Ac}$ were derived shortly after by combining experimental data on ${ }^{227} \mathrm{Ac}^{+}[13,14]$ with input from atomic calculations. Regarding the neutron-deficient isotopes, decay spectroscopy was performed to determine half-lives, $\alpha$-decay energies, and branching ratios [15]. The $g$ factor of ${ }^{217} \mathrm{Ac}$ was measured by using the differential perturbed angular distribution (DPAD) method [11], also used for the high-spin isomer in ${ }^{215} \mathrm{Ac}$ [10]. It was not until 15 years ago that the first ionization potential of actinium was measured [16]. More recently, off-line studies using the long-lived ${ }^{227} \mathrm{Ac}$ isotope confirmed and refined the ionization potential and also looked for efficient laser ionization schemes [17].

This paper reports on the laser ionization and spectroscopy measurements of neutron-deficient ${ }^{212-215} \mathrm{Ac}$ isotopes to study ground-state magnetic-dipole moments, electric-quadrupole moments, and nuclear spins. Part of the results have been reported recently [18]. The paper is structured in the following way: Section II describes in detail the experimental procedure to produce and detect the actinium isotopes. In Sec. III, the calculated efficiencies for both in-gas-jet and the ingas-cell methods are compared. Detailed description of the obtained hyperfine spectra for the in-gas-jet and the in-gascell spectroscopy methods are presented and the different broadening mechanisms affecting the spectral lineshapes and widths are discussed. Finally, in Sec. IV, the extracted magnetic-dipole moments, electric-quadrupole moments, and nuclear-spin assignments are presented and compared with large-scale nuclear-shell-model calculations.

\section{EXPERIMENTAL DETAILS}

In-gas laser ionization and spectroscopy (IGLIS) experiments were performed at the LISOL facility coupled to the cyclotron accelerator of the "Centre de Recherche du Cyclotron" (Cyclotron Research Centre; Louvain-la-Neuve, Belgium) [19]. Both in-gas-cell and in-gas-jet laser spectroscopy experiments were performed on a number of neutron-deficient actinium isotopes. By using the in-gas-cell method, the ${ }^{212-215} \mathrm{Ac}$ isotopes were studied whereas the in-gas-jet method was 


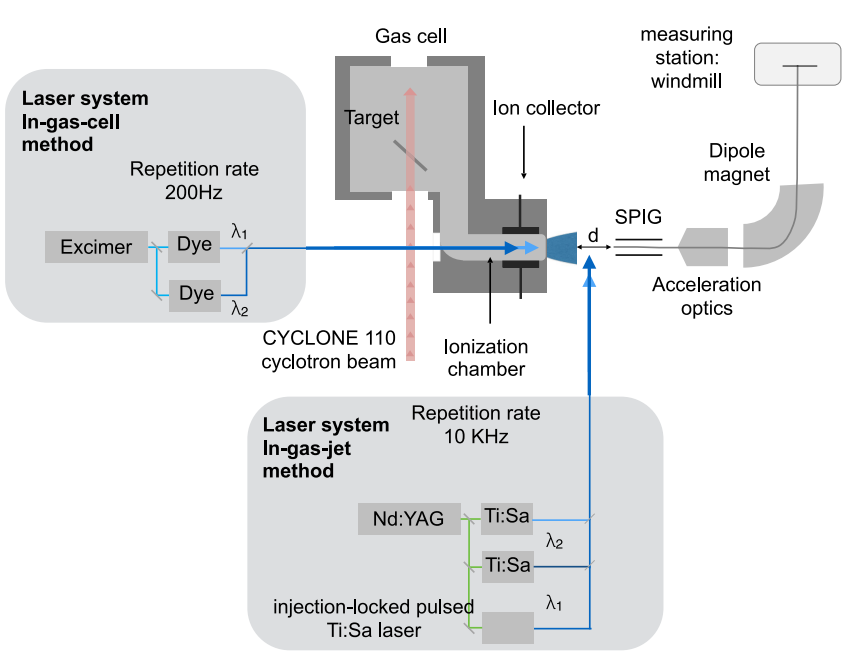

FIG. 1. A schematic representation of the general setup for the IGLIS technique. The distance $d$ from the exit of the gas cell to the SPIG for the in-gas-cell method is $d \sim 2 \mathrm{~mm}$ and for the in-gas-jet method is $d \sim 12 \mathrm{~mm}$.

only applied to ${ }^{214,215} \mathrm{Ac}$. The corresponding half-lives of the actinium isotopes are $T_{1 / 2}\left({ }^{212} \mathrm{Ac}\right)=0.93(5) \mathrm{s}, T_{1 / 2}\left({ }^{213} \mathrm{Ac}\right)=$ $0.738(16) \mathrm{s}, T_{1 / 2}\left({ }^{214} \mathrm{Ac}\right)=8.2(2) \mathrm{s}$, and $T_{1 / 2}\left({ }^{215} \mathrm{Ac}\right)=0.17(1)$ $\mathrm{s}$ [9]. The isotopes of interest were produced in the heavy-ion fusion-evaporation reactions ${ }^{197} \mathrm{Au}\left({ }^{20} \mathrm{Ne}, 4-5 n\right){ }^{212,213} \mathrm{Ac}$ and $\left.{ }^{197} \mathrm{Au}\left({ }^{22} \mathrm{Ne}, 4-5 n\right)\right)^{214,215} \mathrm{Ac}$. The ${ }^{20,22} \mathrm{Ne}$ primary beam with an intensity of 0.16 particle $\mu A$ was sent onto the $1-\mu \mathrm{m}$-thin ${ }^{197} \mathrm{Au}$ target. The target was positioned in the dual gas cell chamber [20] with an inclination angle of $20^{\circ}$ with respect to the normal axis of the primary beam axis; see Fig. 1. The ${ }^{20,22} \mathrm{Ne}$ beam energy, 145 and $143 \mathrm{MeV}$ respectively, was reduced by the entrance window of the gas chamber, the gas cell window [21], and the buffer gas (360 mbar argon). For ${ }^{22} \mathrm{Ne}$, this resulted in a beam energy of $106 \mathrm{MeV}$ in front of the target. The beam energy was chosen in such a way that, for every beam-target combination used, the production cross sections for all studied isotopes were similar. The combined production cross sections for ${ }^{212,213} \mathrm{Ac}$ and ${ }^{214,215} \mathrm{Ac}$ are 2.3 and $2.0 \mathrm{mb}$ [22,23], respectively. The number of ${ }^{215} \mathrm{Ac}$ atoms produced in the target and recoiling out of it was evaluated by taking into account both the $5 \mathrm{MeV}$ energy loss of the ${ }^{22} \mathrm{Ne}$ beam through the target thickness and the energy dependence on the production cross section. This resulted, in the case of ${ }^{215} \mathrm{Ac}$, in a yield of $Y_{\text {recoil }}=3.84 \times 10^{4}$ atoms per particle $\mu \mathrm{C}$ of primary beam recoiling out of the ${ }^{197} \mathrm{Au}$ target.

The reaction products that recoil out of the target were thermalized and neutralized in the buffer gas and flowed toward the exit of the gas cell. Just before the exit hole, an ion collector was installed to suppress the reactions products that were not neutralized. In the case of the in-gas-cell experiments, an exit hole with $1 \mathrm{~mm}$ diameter gave rise to a freely expanding jet. Just before the atoms left the gas cell through the exit hole, they were ionized by using a two-step laser ionization scheme. After leaving the gas cell, the ions were guided by a sextupole ion guide (SPIG) [24] positioned $2 \mathrm{~mm}$ from the exit hole. Between the exit hole and the SPIG, a voltage was applied

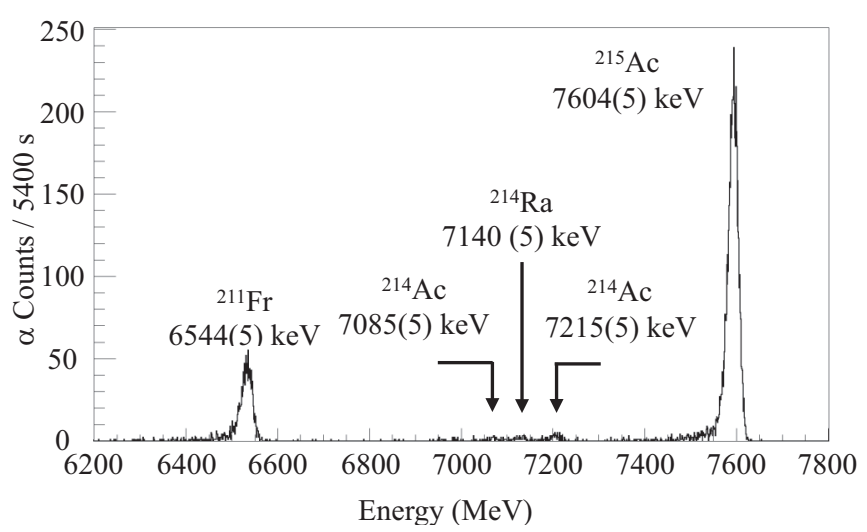

FIG. 2. Alpha spectra of ${ }^{215}$ Ac. The corresponding $\alpha$ energies given were extracted from the National Nuclear Data Center data base (NNDC) [9]. The count rate corresponds to the total number of $\alpha$ particles detected during a full HFS scan.

to dissociate possible molecular ions [20]. For the in-gas-jet experiments, the exit hole was replaced by a de Laval nozzle with a throat diameter of $1 \mathrm{~mm}$ and exit diameter of $4.7 \mathrm{~mm}$ that resulted in a Mach number of $\sim 6$ for the supersonic flow. The distance between the exit of the de Laval nozzle and the SPIG rods was increased to $12 \mathrm{~mm}$ in these experiments to allow the formation of the supersonic jet, as shown in Fig. 1 and discussed in Ref. [25]. Owing to this large distance, the dissociation field was smaller. Because of technical constraints in the LISOL setup, the laser beams were sent perpendicular to the gas jet only irradiating $4 \mathrm{~mm}$ out of the total $12 \mathrm{~mm}$ of the jet. As in the case of in-gas-cell experiments, the ions were guided by the SPIG toward an extraction electrode, accelerated up to $40 \mathrm{keV}$ and sent to the dipole magnet of the LISOL facility. After mass separation, the ions were further transported towards a windmill detection setup [26]. Here, the $\alpha$ decay of the actinium isotopes of interest could be registered by two silicon detectors placed in close geometry at the implantation point that yielded a combined detection efficiency of $24 \%$. The $\alpha$ spectrum of ${ }^{215} \mathrm{Ac}$ is shown in Fig. 2. Finally, by recording the number of $\alpha$ counts as a function of the laser frequency for the first step laser, optical HFS spectra were obtained.

\section{A. Stopping and gas-flow calculation}

In the following sections, the case of ${ }^{215} \mathrm{Ac}$ will be used to discuss the performance of the IGLIS setup. This choice is related to the precisely known cross section, its short half-life (170 ms), and its nuclear-physics interest. Using the $106 \mathrm{MeV}$ energy, calculated by using the Stopping and Range of Ions in Matter (SRIM) [27], of the ${ }^{22} \mathrm{Ne}$ beam entering the ${ }^{197} \mathrm{Au}$ target with a beam-spot diameter of $6 \mathrm{~mm}$, the energy and spatial distribution of the ${ }^{215}$ Ac nuclei recoiling out of the target were also calculated by using SRIM. The same code was used to calculate the spatial distribution of the nuclei stopped in the 350 mbar argon gas. From these simulations, $100 \%$ of the recoiled ions were found to be stopped in the buffer gas. To evaluate the transport efficiency towards the exit hole of the gas cell, the stopping volume was discretized. For every 


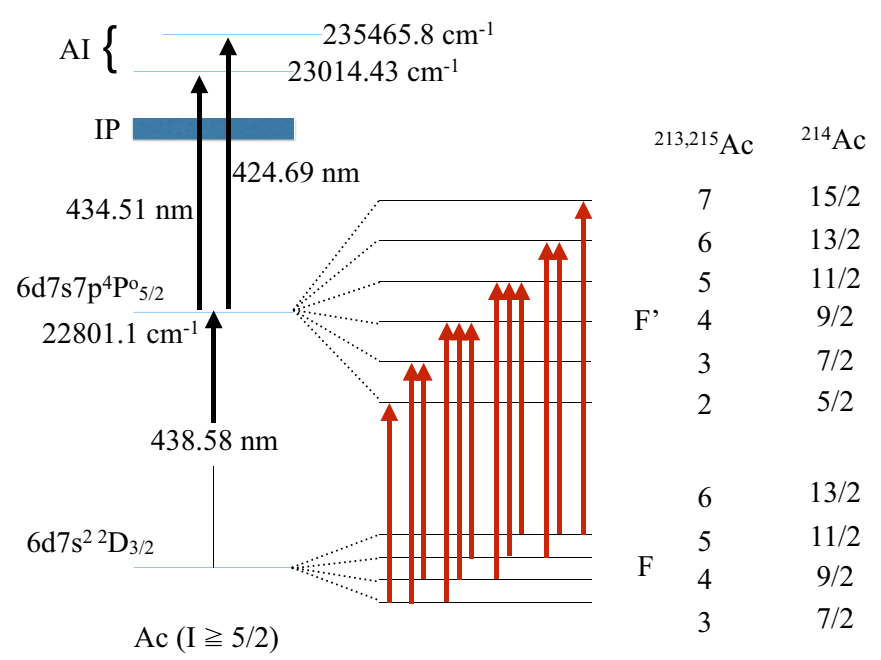

FIG. 3. Schematic representation of the electronic hyperfine structure for the odd-odd and odd-even nuclei investigated in the different experimental campaigns. Notice that the splittings of the ground and excited states are not to scale and the transitions wavelengths are given for vacuum.

subvolume the evacuation path and the evacuation time inside the gas cell were simulated by using the COMSOL multiphysics software [28]. In total, $65 \%$ of the ${ }^{215}$ Ac nuclei reached the exit of the gas cell. This means that $35 \%$ of the ${ }^{215} \mathrm{Ac}$ made a wall collision and are considered as lost. However, $17 \%$ of the nuclei reaching the exit zone have already decayed $(170 \mathrm{~ms})$. For the ${ }^{212,213,214} \mathrm{Ac}$ cases the losses reduce to $0.4 \%, 4.2 \%$, and $3.4 \%$, respectively, owing to their long half-lives. The concept of the mean evacuation time of the gas cell is not useful in this context because the path and its related transition time in the dual gas chamber is strongly dependent on its starting point [29]. In total, $54 \%$ of the ${ }^{215} \mathrm{Ac}$ atoms recoiling out of the target reach the exit hole and could be irradiated by the laser light in the gas cell or in the gas jet.

\section{B. Laser ionization scheme and laser system}

In this study, the $438.58 \mathrm{~nm}$ transition from the $6 d^{1} 7 s^{2}$ atomic ground-state $(J=3 / 2)$ to the $6 d^{1} 7 s^{1} 7 p^{1}$ excited state $(J=5 / 2)$ was used as the first excitation step while the $434.51 \mathrm{~nm}$ transition was used as the second step for the ionization of the excited atoms via an auto-ionizing state, as shown in Fig. 3. The excited atoms were ionized via a second auto-ionizing state with a wavelength of $424.69 \mathrm{~nm}$ $[25,30]$ in the in-gas-jet experiments. In Fig. 3 the allowed atomic transitions which constitute the observed HFS spectrum are shown. More details on the actinium atomic levels and ionization schemes are presented in Ref. [31].

The laser system used for the in-gas-cell laser spectroscopy experiments consists of two dye lasers (Scanmate, Lambda Physik) pumped by one excimer laser (LPX 240i, Lambda Physik), with an average energy per pulse of up to $50 \mathrm{~mJ}$ and a maximum repetition rate of $200 \mathrm{~Hz}$. The two dye laser beams irradiated the gas cell volume just before the exit hole in a longitudinal configuration with respect to the gas flow propagation direction (see Fig. 1). The bandwidth of the first step laser was reduced by an intracavity etalon to around 1.2 $\mathrm{GHz}$. The laser system was operated at a repetition rate of 100 $\mathrm{Hz}$ with typical energies per pulse of 4 and $430 \mu \mathrm{J}$ for the first- and second-step lasers, respectively. The length of the laser pulse was $15 \mathrm{~ns}$. The scanned wavelength was recorded by using an LM-007 (ATOS) wavelength meter. More details on the laser system are given in Ref. [32]. For the in-gas-jet experiments due to the supersonic expansion of the isotopes of actinium, a high-repetition-rate laser system was used to increase the duty cycle. A Nd:YAG laser with a maximum repetition rate of $10 \mathrm{kHz}$ (DM-YAG 60-532, Photonics Industries, Inc.) pumped three Ti:Sa lasers. One of the Ti:Sa lasers featured a bow-tie cavity and was injection-locked by a continuous-wave diode laser resulting in pulsed laser radiation with a narrow spectral bandwidth of about $13 \mathrm{MHz}$ ( $35 \mathrm{~ns}$ pulse width) [31]. The other two lasers used for the ionization step were standard cavities with a Z-shaped resonator featuring a spectral bandwidth of about $3 \mathrm{GHz}$ [33]. The laser energy used for the scans of the HFS was $0.8 \mu \mathrm{J}$ per pulse. The scanned wavelength for the in-gas-jet experiments was recorded with a WS7 (HighFinesse GmbH) wavelength meter.

\section{RESULTS}

\section{A. The efficiency of in-gas laser ionization and spectroscopy}

To characterize the performance of the IGLIS setup, the concept of the IGLIS efficiency $\left(\epsilon_{\text {IGLIS }}\right)$ is defined as the ratio between the ${ }^{215} \mathrm{Ac}$ yield in atoms $/(p \mu \mathrm{C})$ entering the SPIG to that recoiling out of the target $\left[Y_{\text {recoil }}=3.84 \times 10^{4}\right.$ atoms $/(p \mu \mathrm{C})]$. $Y_{\text {IGLIS }}$ is calculated from the number of $\alpha$ decays recorded at the windmill detector setup, corrected for the $\alpha$-detectors efficiency $\left[\epsilon_{\alpha}=24(2) \%\right]$, the $\alpha$ branching ratio $\left(b_{\alpha}\right) 99.91(2) \%$, and the measured transport efficiency though the SPIG and the mass separator $\left[\epsilon_{\text {trans }}=60(10) \%\right]$. In spectroscopy mode, this means with the first-step laser in narrowband mode (i.e., $1.2 \mathrm{GHz}$ for the dye laser and $13 \mathrm{MHz}$ for the Ti:Sa laser) and its energy per pulse limited to $4 \mu \mathrm{J}$ for the dye laser and $0.8 \mu \mathrm{J}$ for the Ti:Sa laser, the IGLIS efficiency $\epsilon_{\text {IGLIS }}$ is found to be $0.42(13) \%$ for the in-gas-cell experiment and $0.40(13) \%$ for the in-gas-jet experiment. In production mode, the efficiency increases with a factor of 1.4(1) when switching to broadband mode and with an additional factor of 1.9(2) when the energy per pulse is increased to $9 \mu \mathrm{J}$ (values from the in-gas-jet experiment). Although the efficiency in both experimental approaches are identical, the in-gas-jet method is superior in contrast to the in-gas-cell method, where $100 \mathrm{~Hz}$ repetition rate of the dye lasers is sufficient to have a $100 \%$ temporal overlap. The $10 \mathrm{kHz}$ repetition rate of the Ti:Sa lasers is not sufficient. The loss factor due to the imperfect temporal overlap can be estimated from the total length of the ionization region for a given repetition rate $x$, which for a $100 \%$ duty cycle should be $x=v / f$, where $v$ is the velocity of the atoms in the gas jet and $f$ is the repetition rate of the laser. For an elongated and homogeneous jet, a repetition rate of $f=10 \mathrm{kHz}$ and a velocity of $v=550 \mathrm{~m} / \mathrm{s}$ (for the gas cell temperature of $300 \mathrm{~K}$ and Mach number 6), the total ionization region should then be $55 \mathrm{~mm}$ [8]. Owing to technical constraints in the current experimental setup only $4 \mathrm{~mm}$ of 
the gas jet were irradiated by both lasers. By replacing the laser beam spots with laser sheets $55 \mathrm{~mm}$ long, optimizing thus the duty cycle, an increase in efficiency by a factor $55 \mathrm{~mm} / 4 \mathrm{~mm} \approx 14$ could have been obtained leading to an expected efficiency for the in-gas-jet method of $5.6 \%$ (see Ref. [25]). In optimal production mode for the in-gas-jet, this leads to $\epsilon_{\text {IGLIS }}$ around $14.9 \%$ and $Y_{\text {IGLIS }}=5.67 \times 10^{3}$ atoms $/(p \mu \mathrm{C})$. This has to be compared with the yield of ${ }^{215} \mathrm{Ac}$ arriving in the laser interaction zone, i.e., $54 \%$ of $Y_{\text {recoil }}$. Thus $\sim 28 \%$ of those ${ }^{215}$ Ac are laser ionized. There are a number of loss factors which are not yet taken into account and could explain this low percentage. By switching off the laser and the ion collector, one can measure the amount of ${ }^{215} \mathrm{Ac}$ nuclei injected in ionic form into the SPIG surviving neutralization. This amounts to $5.7 \%$. Also by setting the mass separator to mass 231 , the $\alpha$ decay of ${ }^{215}$ Ac is observed, proving that ${ }^{215} \mathrm{AcO}^{+}$molecules were not fully cracked by the dissociation voltage. This intensity of the ${ }^{215} \mathrm{AcO}^{+}$ions is $\sim 1 / 3$ of the intensity of the ${ }^{215} \mathrm{Ac}$ ions, when lasers and ion collector are switched off. The formation of molecules and clusters are a well-known phenomenon in the high-density regime of the gas cell and are strongly sensitive to the purity of the gas. In Ref. [25] it is shown that, even in the gas jet, photoions can form clusters with argon (and possibly nitrogen). The total amount of neutralization-surviving ions will probably be larger than $7.6 \%$ because no other molecular sidebands have been investigated. Next to the charged molecules and clusters containing ${ }^{215} \mathrm{Ac}$, also neutral ones will be present in the gas cell and gas jet and will be lost for laser ionization. The IGLIS efficiency in the gas cell rises in the production mode to $1.1 \%$, a factor of 13.3 lower than in the gas jet with optimal time overlap. This loss factor to neutralization-surviving ions and to molecules and clusters is essentially the same but now photoions, produced before the ion collector (see Fig. 1), are lost. Furthermore, the high density can also lead to collisional deexcitation of the intermediated atomic level, inhibiting the ionization step. The mean time between collisions is $1.7 \mathrm{~ns}$ for the gas cell and $200 \mathrm{~ns}$ in the gas jet for a Mach number of 6 . These times have to be compared with the pulse lengths of the lasers: $15 \mathrm{~ns}$ for the dye lasers used in the gas cell and $35 \mathrm{~ns}$ for the Ti:Sa lasers used in the gas jet.

\section{B. Selectivity}

The selectivity in the spectroscopy mode is defined as the ratio between the $\alpha$ counts $/ 50 \mathrm{~s}$ obtained in the maximum of the strongest resonance to the fitted background count rate. For the in-gas-cell method, this results in a value of 8.3(17) while for the in-gas-jet method, a value of 121(27) was obtained. In the production mode, the selectivity is higher. In optimal conditions with respect to the efficiency, the selectivity of the in-gas-jet technique should increase to above 3000 [18].

\section{In-gas-cell spectroscopy}

All HFS data were analyzed by using the Statistical Analysis Toolbox for Laser Spectroscopy (SATLAS) [34]. This python package enables the fitting of Voigt profiles to the hyperfine spectra by using either a minimization by a nonlinear least squares method or by maximum likelihood optimization. Both methods were tested, showing consistent results within the statistical errors. For the gas-cell data, shown in Fig. 4, only the excited state was resolved. The hyperfine splitting of the electronic ground state was not resolved due to the presence of collisional broadening (see Sec. IIIE). For the analysis of the in-gas-cell data, the ratio of the magnetic hyperfine coupling constant of the upper (excited) state, $a_{u}$, to that of the lower (ground) state, $a_{l}$, was fixed to the values extracted for ${ }^{227} \mathrm{Ac}$, for which $a_{u} / a_{l}=41.6(8)(16)$ [31]. The error in the first bracket corresponds to the statistical error and the second value in brackets corresponds to the systematic error (see Sec. III F). This ratio is isotope independent in the absence of a hyperfine anomaly. The quadrupole hyperfine coupling constants were fixed to zero. The in-gas-jet data obtained for ${ }^{214,215}$ Ac allowed an analysis of the reliability of the fit of the low resolution in-gas-cell data by using the saturated electronic transitions (see Sec. III D). The contribution of the Doppler broadening stemming from thermal atomic motion in addition to the laser bandwidth was calculated and resulted in 1.4(1) $\mathrm{GHz}[8,18]$. This Gaussian contribution to the total full-width at half maximum (FWHM) was fixed in the Voigt fits while the Lorentzian contribution was unconstrained. The average FWHM of the spectral lines, extracted from the Voigt fits, was 5.8(3) GHz.

\section{The in-gas-jet spectroscopy data}

The hyperfine spectrum measured for ${ }^{214}$ Ac obtained with the in-gas-jet spectroscopy is shown in Fig. 5 whereas the HFS of ${ }^{215} \mathrm{Ac}$ can be found in Ref. [18]. The enhanced resolution of the method allowed to resolve the ground-state hyperfine splitting and to determine the electric hyperfine coupling constant, $b_{l}$, for the ground-state as the contribution of the $b_{u}$ constant to the splitting of the excited state is much smaller. In Fig. 5(b) the high-energy doublet in ${ }^{214} \mathrm{Ac}\left(F_{1}=11 / 2 \rightarrow\right.$ $F_{2}=13 / 2$ and $F_{1}=13 / 2 \rightarrow F_{2}=13 / 2$ ) is shown. A total FWHM of 390(20) MHz was obtained. The fitting of the HFS was performed with unconstrained transition amplitudes and fixed ratios of the magnetic and electric coupling constants to the ratios extracted from off-line measurements on ${ }^{227} \mathrm{Ac}[31]$ : $a_{u} / a_{l}=41.6(8)(16)$ and $b_{u} / b_{l}=0.184(6)(8)$ (see Table I). The data were also fit with $a_{u}$ and $a_{l}$ unconstrained. Within the statistical uncertainty, this had no effect on the deduced $a_{u}$ but resulted in slightly different ratios of $a_{u} / a_{l}$ of 44.8(19)(32) and 45.6(25)(35) for ${ }^{214,215} \mathrm{Ac}$, respectively. This might indicate the presence of a hyperfine anomaly. However, the effect is smaller than the measured systematic uncertainty, preventing any firm conclusion (see Sec. III F).

The in-gas-jet data were also used to determine an optimal fitting procedure for the spectra from the gas-cell data. Two different fitting procedures were used. In the first method the relative amplitudes were fixed for those transitions sharing the same final state, to the ratio given by the Racah coefficient [35]. The second procedure uses a saturation parameter to take into account the influence of the laser power in the transition amplitudes [35]. The extracted values of the hyperfine coupling constants for ${ }^{214,215}$ Ac were the same for both procedures within the statistical errors. We used the latter procedure to 

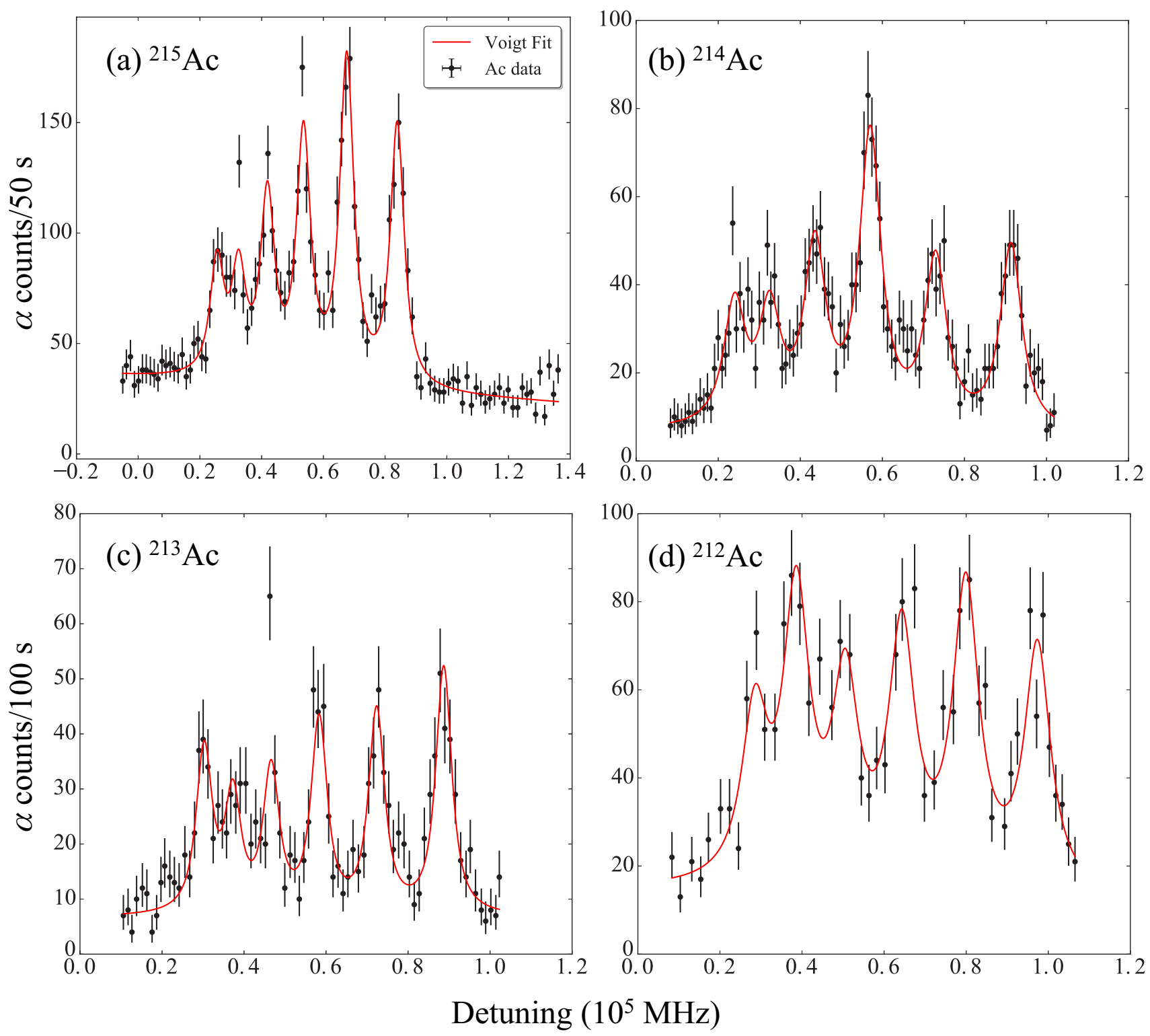

FIG. 4. Hyperfine structure of (a) ${ }^{215} \mathrm{Ac}$, (b) ${ }^{214} \mathrm{Ac}$, (c) ${ }^{213} \mathrm{Ac}$, and (d) ${ }^{212}$ Ac measured by using the in-gas-cell method. The $x$ axes denote the detuning, $w_{0}-w$, where $w_{0}$ is the center of gravity for ${ }^{227} \mathrm{Ac}$.

extract the hyperfine magnetic coupling constants from the in-gas-cell data, as reported in Table I.

\section{E. Collisional broadening and shift}

In the gas-cell experiments, the dominant line broadening mechanism is due to collisions of actinium with the argon atoms [8]. This interaction also results in a frequency shift of the spectral lines. The shift is positive if the interaction between the atomic species is repulsive and negative if the interaction is attractive [36]. The collisional broadening coefficient $\gamma_{\text {coll }}$ was extracted from several scans of the high-energy singlet in ${ }^{215} \mathrm{Ac}$ for different buffer-gas pressures (see Fig. 6). This resulted in a collisional broadening coefficient $\gamma_{\text {coll }}=11.5(10)$ $\mathrm{MHz} / \mathrm{mbar}$ and in a collisional shift coefficient $\Delta_{\mathrm{sh}}=-3.7(9)$ $\mathrm{MHz} / \mathrm{mbar}$, corresponding to an attractive interaction between the atomic species.
The ratio between the coefficients $\gamma_{\text {coll }}$ and $\Delta_{\text {sh }}$ determines the type of interaction between the atomic species. In the case of a van der Waals interaction, the theoretical ratio is $\gamma_{\text {coll }} / \Delta_{\mathrm{sh}}=2.76$ [36]. Values of this ratio studied for different elements in argon gas at LISOL were compared with the actinium results (see Table II). The results, within experimental uncertainties, show the same type of interaction potential of these elements with the argon buffer gas. The case for ${ }^{58} \mathrm{Ni}$ needs to be investigated once more to confirm a real deviation from such a ratio. The total uncertainty in the ratios is dominated by the error in extracting the Lorentzian widths.

\section{F. Systematic uncertainties}

The measured hyperfine spectra are subject to a series of systematic uncertainties, dominated by the different wave- 


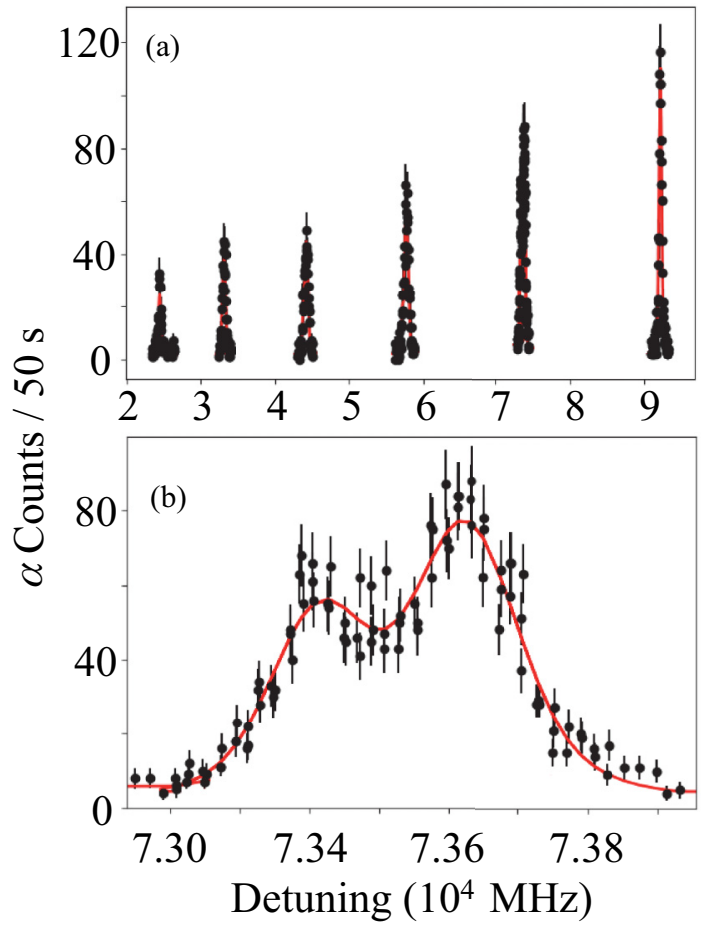

FIG. 5. (a) Atomic hyperfine structure of ${ }^{214} \mathrm{Ac}$ measured by using the in-gas-jet method. In red is the best fit using Voigt profiles to the experimental data. (b) High-energy doublet of ${ }^{214} \mathrm{Ac}$, corresponding to the $F=11 / 2 \rightarrow F^{\prime}=13 / 2$ and $F=13 / 2 \rightarrow$ $F^{\prime}=13 / 2$ transitions. A FWHM of 320(20) MHz was obtained. The energy per pulse for this scan was $0.8 \mu \mathrm{J}$. The $x$ axis denotes the detuning, $w_{0}-w$, where $w_{0}$ is the center of gravity for ${ }^{227} \mathrm{Ac}$. The large hyperfine splitting between multiplets corresponds to the splitting of the excited atomic state and is dominated by the magnetic hyperfine coupling constant $a_{u}$.

length meters used and their precision [18]. During the five days experimental campaign devoted to the in-gas-jet laser spectroscopy studies, the high-energy singlet of ${ }^{215} \mathrm{Ac}$ ( $F=6 \rightarrow F^{\prime}=7$ in Fig. 3) was repeatedly scanned. From

TABLE I. Spin $I$ and hyperfine coupling constants $a$ and $b$ for the $22801.1 \mathrm{~cm}^{-1}$ energy level and for the ground state, respectively, extracted from the in-gas-cell and the in-gas-jet experiments. The first parentheses in the reported data indicate the statistical error. The second parentheses correspond to the systematic error. The number of scans available for the statistical analysis is listed in the last column.

\begin{tabular}{lcccc}
\hline \hline Isotope & $I$ & $\mathrm{a}_{u}(\mathrm{MHz})$ & $b_{l}(\mathrm{MHz})$ & No. of scans \\
\hline Gas jet & & & & \\
${ }^{215} \mathrm{Ac}$ & $9 / 2$ & $2377.0(10)(40)$ & $13(26)(20)$ & 1 \\
${ }^{214} \mathrm{Ac}$ & 5 & $2498.4(10)(40)$ & $48(22)(20)$ & 1 \\
\hline Gas cell & & & & 3 \\
${ }^{215} \mathrm{Ac}$ & $9 / 2$ & $2386(17)(90)$ & & 2 \\
${ }^{214} \mathrm{Ac}$ & $(5)$ & $2525(22)(90)$ & & 3 \\
${ }^{213} \mathrm{Ac}$ & $(9 / 2)$ & $2385(31)(90)$ & & \\
${ }^{212} \mathrm{Ac}$ & $(7)$ & $1837(22)(90)$ & & \\
\hline Off-line & & & & \\
${ }^{227} \mathrm{Ac}$ & $3 / 2$ & $2104.8(10)(20)$ & $597(4)(5)$ & \\
\hline \hline
\end{tabular}

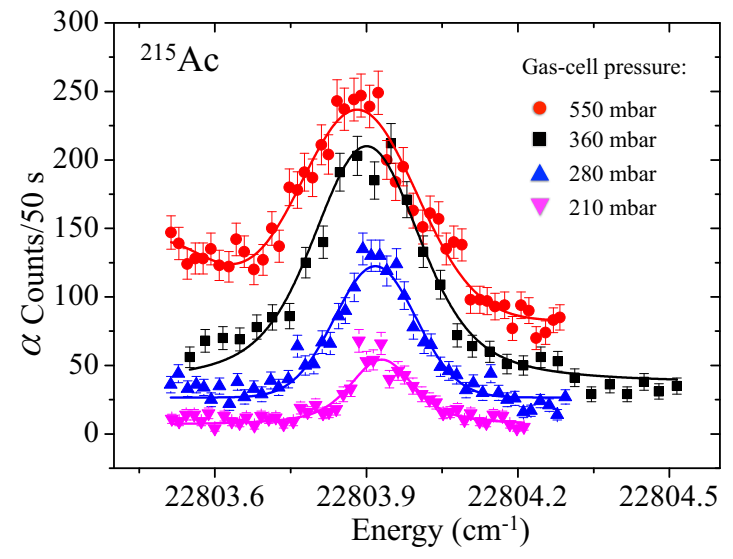

FIG. 6. Comparison of the collisional broadening and the collisional shift as a function of argon pressure inside the gas cell for the $F=6 \rightarrow F^{\prime}=7$ transition. The doublet $\left(F=5 \rightarrow F^{\prime}=6\right.$ and $F=6 \rightarrow F^{\prime}=6$; see Fig. 3 ) was included in the fit due to its presence in the 550 mbar scan.

these measurements, a standard deviation of $30 \mathrm{MHz}$ on the centroid of the resonance was found. To estimate the impact of this uncertainty on the deduced hyperfine coupling constants $a$ and $b$, the HFS were simulated with the position of the different resonances varied randomly by $30 \mathrm{MHz}$. The scattering in the position of the peaks has an impact of maximally 4 and $20 \mathrm{MHz}$ in the hyperfine coupling constants $a$ and $b$, respectively, which are taken as the systematic uncertainty (see Table I). In the case of the in-gas-cell data, a systematic error of $90 \mathrm{MHz}$ was obtained for the hyperfine coupling constant $a$. This uncertainty originates from the multimode nature of the laser light used in the experiment [6].

\section{G. Saturation of the spectral lines in the in-gas-jet experiment}

The Lorentzian broadening in the spectra obtained with the in-gas-jet method was found to be caused by atom-atom collision and power effects [8]. To determine possible residual collisional broadening, we measured the high-energy singlet and doublet in ${ }^{215} \mathrm{Ac}$ for different pulse energies of the first excitation step. The results of the measurements for the high-energy singlet are shown in Fig. 7. These scans make it possible to extract a collisional broadening contribution of $\Gamma_{\text {coll }}^{\mathrm{exp}}=42(6) \mathrm{MHz}$ from the extrapolation to the zero-energyper-pulse value. The total Gaussian broadening of 280(30)

TABLE II. Comparison between collisional broadening $\gamma_{\text {coll }}$ and shift $\Delta_{\text {sh }}$ when the studied isotope interacts with argon atoms used as buffer gas. The reported $\gamma_{\text {coll }}$ and $\Delta_{\text {sh }}$ values are given in $\mathrm{MHz} / \mathrm{mbar}$. The values for copper, silver, and nickel are taken from Refs. [5], [6], and [37], respectively.

\begin{tabular}{lcccc}
\hline \hline Isotope & $\gamma_{\text {coll }}$ & $\Delta_{\text {sh }}$ & $\gamma_{\text {coll }} / \Delta_{\text {sh }}$ & Transition \\
\hline${ }^{58} \mathrm{Ni}$ & $11.3(6)$ & $-5.5(3)$ & $-2.1(2)$ & $3 d^{8} 4 s^{2} \rightarrow 3 d^{8} 4 s 4 p$ \\
${ }^{63} \mathrm{Cu}$ & $5.4(5)$ & $-1.9(1)$ & $-2.8(5)$ & $3 d^{10} 4 s^{1} \rightarrow 3 d^{9} 4 s 4 p$ \\
${ }^{107} \mathrm{Ag}$ & $12(2)$ & $-3.7(4)$ & $-3.2(5)$ & $4 d^{10} 5 s^{1} \rightarrow 4 d^{10} 5 p$ \\
${ }^{215} \mathrm{Ac}$ & $11.5(10)$ & $-3.7(9)$ & $-3.1(8)$ & $6 d^{1} 7 s^{2} \rightarrow 6 d 7 s 7 p$ \\
\hline \hline
\end{tabular}




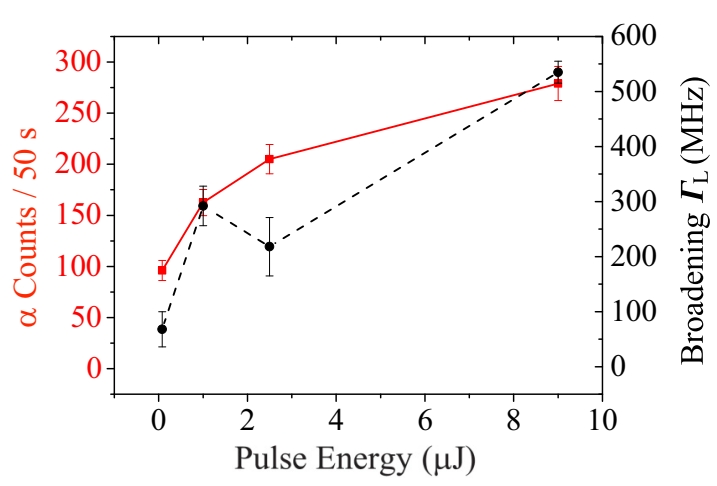

FIG. 7. Left axis, saturation curve for the high-energy singlet $\left(F=6 \rightarrow F^{\prime}=7\right)$ in ${ }^{215} \mathrm{Ac}$ and right axis, evolution of the Lorentzian broadening $\Gamma_{L}$ as a function of the laser energy. The Gaussian component of the total broadening was fixed in the fit of the experimental data. The lines connecting the points are only to guide the eyes.

MHz was obtained from the same fit. A natural linewidth of 4 MHz was obtained in multiconfiguration Dirac-Hartree-Fock (MCDHF) calculations [18]. Simulations performed by using COMSOL indicated a temperature of $30 \mathrm{~K}$ in the jet for our experimental conditions. The Gaussian broadening due to thermal motion in the supersonic jet in these conditions corresponds to $180 \mathrm{MHz}$. The remaining $214 \mathrm{MHz}$ is caused by the divergence of the supersonic jet at the interaction point with the lasers due to a nonoptimal matching of the background and the jet pressure. Based on this temperature value a semi-empirical collisional broadening was calculated by using Eqs. (20) and (22) from Ref. [8]. The calculated value $\Gamma_{\mathrm{coll}}^{\mathrm{cal}}=53(5) \mathrm{MHz}$ is in good agreement with our experimental value. The deviation in the width from the trend observed at $2.5 \mu \mathrm{J}$ in Fig. 7 , might be due to the fact that these data were taken at the beginning of the run during the optimization procedures. The three other data points were taken immediately preceding the spectroscopy scans and thus under stable conditions. Especially, for nonoptimized gas-jet and background-pressure conditions, the overlap between the laser beams and the gas jet can influence the linewidth, as has been recently shown in off-line studies [29].

The different scans of the high-energy doublet of ${ }^{215} \mathrm{Ac}$ are displayed in Fig. 8. The theoretical ratio (from Racah coefficients) between the transitions amplitudes $F_{1}=5 \rightarrow$ $F_{2}=6$ (cyan) and $F_{1}=6 \rightarrow F_{2}=6$ (green) is 2.25 . This ratio is observed only in the scan at $0.08 \mu \mathrm{J}$. The change of this ratio as a function of laser energy can have a significant impact on the model used for the description of the hyperfine structure spectra [38]. Due to the different power-broadening regimes at which each atomic transition is exposed, for an accurate extraction of the broadening mechanism for each atomic line, a more accurate fitting to the experimental data should take into account individual broadening values. This is particularly important for the in-gas-jet spectroscopy. For fitting the spectra at different laser energies, the Gaussian-like broadening was fixed and shared between both resonances, while the Lorentzian broadening was unconstrained. This procedure was tested in the case of the in-gas-jet data and no change in the values of the hyperfine coupling constants was

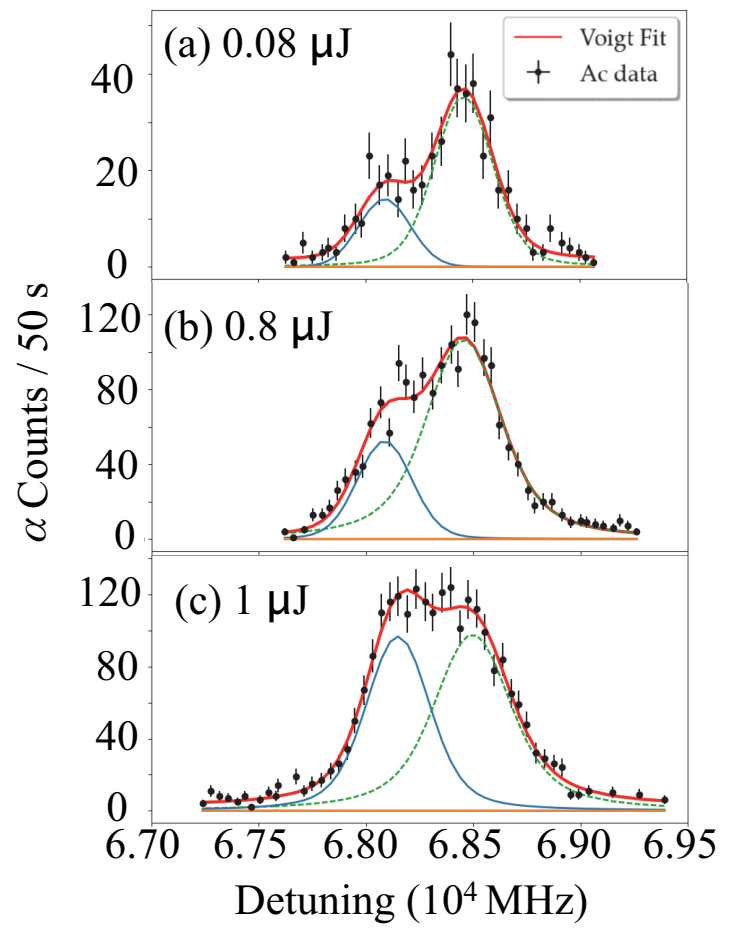

FIG. 8. (a)-(c) Change in the signal amplitude for the transitions $F_{1}=5 \rightarrow F_{2}=6$ (blue) and $F_{1}=6 \rightarrow F_{2}=6$ (dashed green) for different laser energy per pulse in ${ }^{215} \mathrm{Ac}$. The red curve is the total fit to the experimental data. We observed that, due to the different transition strengths, different resonances are exposed to a different power broadening. The $x$ axis denotes the detuning, $w_{0}-w$, where $w_{0}$ is the center of gravity for ${ }^{227} \mathrm{Ac}$.

observed, even when different spectral lines have different values for the broadening in the result of the unconstrained fitting procedure.

In Table I, the extracted hyperfine coupling constants from the different scans are presented.

\section{DISCUSSION}

\section{A. Reference values}

To deduce the nuclear ground-state $g$ factors for ${ }^{212-215} \mathrm{Ac}$ and electric-quadrupole moments $Q$ for ${ }^{214,215} \mathrm{Ac}$ from the extracted hyperfine coupling constants (see Table I), reference isotopes were used as shown in the following equations:

$$
\begin{aligned}
g & =g_{\mathrm{ref}} \frac{a}{a_{\mathrm{ref}}}, \\
Q & =Q_{\mathrm{ref}} \frac{b}{b_{\mathrm{ref}}},
\end{aligned}
$$

where the subindex "ref" indicates the reference values reported either for ${ }^{227} \mathrm{Ac}$ or for the isomeric states in ${ }^{215} \mathrm{Ac}$. Only the $\mu$ and $Q$ values for ${ }^{227}$ Ac obtained from optical spectroscopy are reported: $\mu\left({ }^{227} \mathrm{Ac}\right)=1.1(1) \mu_{N}$ and $Q\left({ }^{227} \mathrm{Ac}\right)=$ $1.7(2) \mathrm{eb}$ [14]. The uncertainties were not justified in the original publication (see Ref. [12]). In Ref. [18] we reported results obtained from atomic calculations using the MCDHF method 
TABLE III. Deduced $g$ factors and quadrupole moments for the actinium isotopes. The $g$ factor for the ${ }^{227} \mathrm{Ac}$ isotope corresponds to the recalculated value when the $g$ factor for ${ }^{215} \mathrm{Ac}$ is used as a reference. The quadrupole moment of ${ }^{227} \mathrm{Ac}$ was used as reference value and was obtained in MCDHF calculations. The brackets around the spin values correspond to a tentative assignment.

\begin{tabular}{lclc}
\hline \hline Isotope & $I$ & \multicolumn{1}{c}{$g$} & $Q(\mathrm{eb})$ \\
\hline${ }^{212} \mathrm{Ac}$ & $(7)$ & $0.711(13)(37)$ & \\
${ }^{213} \mathrm{Ac}$ & $(9 / 2)$ & $0.923(18)(39)$ & \\
${ }^{214} \mathrm{Ac}$ & 5 & $0.967(14)(14)$ & $+0.14(6)(6)$ \\
${ }^{215} \mathrm{Ac}$ & $9 / 2$ & $0.920(13)^{\mathrm{a}}$ & $+0.04(8)(6)$ \\
${ }^{227} \mathrm{Ac}$ & $3 / 2$ & $0.815(12)(12)$ & $+1.74(10)^{\mathrm{b}}$ \\
\hline \hline
\end{tabular}

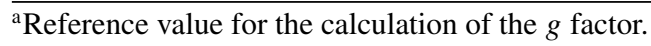

${ }^{\mathrm{b}}$ Reference value for the calculation of the $Q$ moments.

to analyze the atomic structure and to deduce the hyperfine coupling constants from computations of the magnetic-field and electric-field gradients for the experimentally observed transition in ${ }^{227} \mathrm{Ac}$. This resulted in values of $\mu=1.07(18) \mu_{N}$ and $Q=1.74(10)$ eb. The uncertainties on the extracted values reflect the standard deviation of the results from the eight atomic energy levels used to calculate this mean value $[18,39]$. An alternative, nuclear-model-dependent way, to determine $\mu$ for the actinium isotopes is to use the experimental $g$ factors of the high-spin nuclear isomeric states in ${ }^{215} \mathrm{Ac}$ as a reference. The $I=21 / 2^{-}$state belongs to the lowest seniority $v=3$ states in ${ }^{215} \mathrm{Ac}$ and is formed by three $1 h_{9 / 2}$ protons coupling to $\left[(h 9 / 2)^{3}\right]_{21 / 2^{-}}$. A number of experimental and theoretical investigations proved that the admixtures of states belonging to other configurations and to collective excitations of these high-spin states in $N=126$ isotones are small [10,11,40,41]. Thus, the additivity relation (see e.g., Ref. [10]) $g(h 9 / 2)^{1}=$ $g(h 9 / 2)^{3}$ holds for the experimental single-particle $g$ factor of the $1 h_{9 / 2}$ proton in these isotones. This is evident in the case of ${ }^{213} \mathrm{Fr}$, where the isomeric states with spin $I=17 / 2^{-}$ and $I=21 / 2^{-}$, both with the nuclear configuration $\left(\pi h_{9 / 2}\right)^{3}$, exhibit the same $g$ factor as the nuclear ground state, $g(I=$ $9 / 2)=0.89(2), g(I=17 / 2)=0.88(20)$, and $g(I=21 / 2)=$ $0.888(3)$ [9]. For ${ }^{215} \mathrm{Ac}$ the $g$ factors for the isomeric states with nuclear spin $I=17 / 2$ and $I=21 / 2$ were deduced from $\gamma$-ray spectroscopy to be the same and correspond to $g=0.920$ (19) [10]. These $g$ factors were corrected for the Knight shift and diamagnetic shielding [11,42]. Following the aforementioned procedure, we assign the weighted average value of the $g$ factor of the two isomers in ${ }^{215} \mathrm{Ac} g=0.920(13)$ as the $g$ factor for the ground state of ${ }^{215} \mathrm{Ac}$ and use this as a reference value for ${ }^{212-214} \mathrm{Ac}$ and ${ }^{227} \mathrm{Ac}$ isotopes in Eq. (1) (see Table III). Compared with the values deduced by using the MCDHF calculations, the $g$ factors are $20 \%$ larger but consistent with the theoretical values given their uncertainty of $17 \%$. The $Q$ values of the neutron-deficient actinium isotopes were deduced by using a reference $Q{ }^{227} \mathrm{Ac}$ ) value derived from MCDHF calculations. Table III summarizes the results for the $g$ factors, $Q$ values, and nuclear spins from this work.
TABLE IV. Additivity rule $g$-factor value for two different nuclear configurations, $g_{\text {add }}[46]$, in comparison with the $g$ factor extracted from the HFS from ${ }^{212} \mathrm{Ac}$ when the indicated nuclear spin is used to fit the experimental data. The proton $g$ factor was taken from ${ }^{215} \mathrm{Ac}$ (see Table III). The neutron $p_{1 / 2} g$ factor was taken from ${ }^{213} \mathrm{Ra}$ [48] and the $f_{5 / 2} g$ factor from ${ }^{211} \mathrm{Ra}[9]$.

\begin{tabular}{lllc}
\hline \hline Configuration & $I$ & $g_{\text {add }}$ & $g$ \\
\hline$\left(\pi h_{9 / 2}\right)\left(v f_{5 / 2}\right)$ & 7 & $0.717(9)$ & $0.711(13)$ \\
& 6 & $0.744(9)$ & $0.818(15)$ \\
& 5 & $0.787(11)$ & \\
$\left(\pi h_{9 / 2}\right)\left(v p_{1 / 2}\right)^{-1}$ & 4 & $0.863(12)$ & \\
& 5 & $0.950(12)$ & \\
\hline \hline
\end{tabular}

\section{B. Spin assignments}

The HFS measurements were used finally to determine the ground-state $g$ factor, the $Q$ value, the nuclear spin $I$, and the difference in mean-square nuclear charge radii of the actinium isotopes. The analysis of the mean-square nuclear charge radii will be given in a separate presentation. The energy of the individual hyperfine levels is given by

$$
E_{\mathrm{HF}}=\frac{a}{2} C+\frac{b}{4} \frac{3 C(C+1)-2 I(I+1) 2 J(J+1)}{2 I(2 I-1) 2 J(2 J-1)},
$$

with $a$ and $b$ being the magnetic-dipole and electricquadrupole hyperfine coupling constants, respectively, and $C=F(F+1)-I(I+1)-J(J+1)$ is the Casimir constant in which $J$ is the electronic total angular momentum. The nuclear spins that are not reported in this paper were taken from the National Nuclear Data Center database [9].

$$
\text { 1. }{ }^{215} \mathrm{Ac}
$$

The high-resolution in-gas-jet data enable a firm assignment for the ground-state spin of ${ }^{215} \mathrm{Ac}$ to $I=9 / 2$ because the fit using other assumptions for the nuclear-spin values cannot find the Voigt profile that describes the data and results in a unrealistic $\chi^{2}$. The $I=9 / 2$ nuclear spin assignment supports the unpaired proton in the $\pi 1 h_{9 / 2}$ orbital as the dominant configuration of the ${ }^{215} \mathrm{Ac}$ ground state. The same nuclearspin value was observed for the ground states of the odd-odd isotones in francium $[43,44]$ and bismuth [45].

$$
\text { 2. }{ }^{214} \mathrm{Ac}
$$

For ${ }^{214} \mathrm{Ac}$ a firm assignment of the nuclear ground-state spin of $I=5$ was also possible from the fit because the other spin values resulted in unrealistic $\chi^{2}$ values. This value supports the systematic trend of the nuclear spins in the ${ }^{212} \mathrm{Fr},{ }^{210} \mathrm{At}$, and ${ }^{208} \mathrm{Bi}$ isotones. Moreover, it allows us to identify the dominant nucleon configurations giving rise to the $g$ factor of ${ }^{214} \mathrm{Ac}$ by using the additivity rule [46] (see Table IV). Assuming a $\left[\left(\pi h_{9 / 2}\right)\left(v p_{1 / 2}\right)^{-1}\right]_{I=5}$ configuration for ${ }^{214} \mathrm{Ac}$ in $g\left({ }^{214} \mathrm{Ac}\right)_{\text {add }}=$ $0.950(12)$, in agreement with our experimental value, as shown in Table III. 


$$
\text { 3. }{ }^{212,213} \mathrm{Ac}
$$

Because the ground-state HFS was not resolved in the spectra obtained with the in-gas-cell method, no firm nuclearspin assignment for ${ }^{212,213} \mathrm{Ac}$ could be made from the fit.

A spin and parity assignment of $I=9 / 2$ for the ${ }^{213} \mathrm{Ac}$ ground state was proposed in Ref. [47]. It is based on the consideration of the $\alpha$-decay chain ${ }^{217} \mathrm{~Pa} \rightarrow{ }^{213} \mathrm{Ac} \rightarrow$ ${ }^{209} \mathrm{Fr} \rightarrow{ }^{205} \mathrm{At} \rightarrow{ }^{201} \mathrm{Bi}$. All $\alpha$ decays in this chain were assigned to ground-state to ground-state transitions with the $\alpha$-hindrance factors close to unity. Therefore these transitions were regarded as transitions connecting levels of equal spin and parity. Thus, a spin and parity assignment of $I=9 / 2$ for ${ }^{213} \mathrm{Ac}$ was proposed, taking into account corresponding reliable assignments for the ground states of ${ }^{209} \mathrm{Fr},{ }^{205} \mathrm{At}$, and ${ }^{201} \mathrm{Bi}$ [9]. Our HFS data were fit by assuming $I=9 / 2$, but changing the nuclear-spin values for these nuclei by one unit up or down did not significantly change the $\chi^{2}$ value of the fit.

In the case of ${ }^{212} \mathrm{Ac}$, the $\alpha$-decay feeding the ${ }^{208} \mathrm{Fr}$ nuclear ground state suggests a nuclear ground-state spin of $I=7$ [9]. The $I=7$ spin assignment is based on the low hindrance factor $(\mathrm{HF} \sim 2.1$, estimated by using the theoretical branching ratio) for the $\alpha$-decay transition ${ }^{212} \mathrm{Ac} \rightarrow{ }^{208} \mathrm{Fr}$ [7379(8) keV] with the assumption that this is a ground-state to groundstate transition. Correspondingly, ${ }^{212} \mathrm{Ac}$ ground-state spin is supposed to be equal to the firmly established ${ }^{208} \mathrm{Fr}$ groundstate spin $I=7$. The ${ }^{212} \mathrm{Ac}$ data were fit with $I=7$ (see Table I), but $I=6$ could not be excluded. From the quality of the fit spin values $I=5$ and lower could be excluded. It can be shown that only the nuclear configuration $\left[\left(\pi h_{9 / 2}\right)\left(v f_{5 / 2}\right)\right]_{I=7}$ results in good agreement with the experimental value, listed in Table IV. Notice that the fit assuming $I=6$ results in $g=$ $0.818(15)$.

\section{Large-scale nuclear-shell-model calculations}

A comparison of the $g$ factors for ${ }^{212-215} \mathrm{Ac}$ isotopes with the isotones of francium and bismuth is shown in Fig. 9. The data of the bismuth isotopes were extracted from the NNDC [9]. For the francium data, the magnetic-dipole moment value $\mu\left({ }^{210} \mathrm{Fr}\right)=4.35(5) \mu_{N}$ from [49] was used to recalculate the magnetic-dipole moment values for ${ }^{211-213} \mathrm{Fr}$ by using the hyperfine coupling constants reported in Refs. [43,50]. The figure also shows the effective $g$ factor, $g_{\text {eff }}$, calculated by using $g_{\text {eff }}^{s}=0.6 g_{\text {free }}^{s}$ and $g^{l}=1$ in the Schmidt formula [46].

An empirical $g$ factor, $g_{\text {emp }}$, corresponding to the measured $g$ factor for ${ }^{209} \mathrm{Bi}$ [51], is introduced to compare the evaluation of the $g$ factors in this region. A similar trend for the $g$ factors of the even- $N$ isotopes of actinium, francium, and bismuth is observed. The deviation of the $g$ factor for ${ }^{214} \mathrm{Ac}$ compared with francium and bismuth isotones needs to be investigated.

To understand the underlying nuclear structure, large-scale nuclear-shell-model calculations were performed in the region northwest of ${ }^{208} \mathrm{~Pb}$ on the nuclear chart and $\mu$ and $Q$ values were extracted. The valence space used for the calculations was defined as $\pi\left(h_{9 / 2}, f_{7 / 2}, i_{13 / 2}\right)$ and $v\left(f_{5 / 2}, p_{3 / 2}, p_{5 / 2}, i_{13 / 2}\right)$ and was combined with the PBPKH interaction [52] to obtain the nuclear moments. The large numbers of valence particles and orbits in the model space made it necessary to apply a

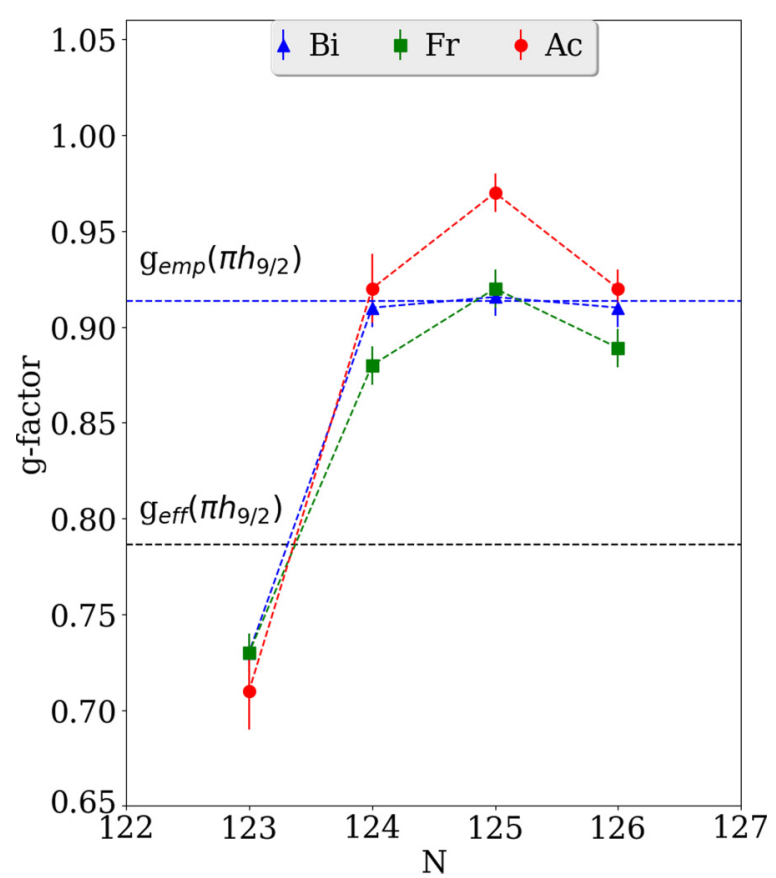

FIG. 9. Comparison between the $g$ factors for the actinium isotopes with isotopes of francium and bismuth. The $g_{\text {eff }}$ is the Schmidt value typical for this region [43]. The blue dashed line corresponds to the empirical $g$-factor value from ${ }^{209} \mathrm{Bi}$ [51]. Here, only the statistical errors are shown.

truncation to make calculations with the OXBASH code [53] feasible (see details in Refs. [1] and [18]). For the actinium isotopes a comparison with the results from the large-scale nuclear-shell-model calculations are shown in Fig. 10. Apart from a deviation in the $g$ factor of ${ }^{214} \mathrm{Ac}$, excellent agreement with the experimental data is obtained. The difference in the shell-model $g$-factor value and the experimental value for the $I^{\pi}=5^{+}$ground state in ${ }^{214} \mathrm{Ac}$ is due to the neutron $v p_{1 / 2}$ in the dominant $\pi\left(h_{9 / 2}^{n} \otimes v p_{1 / 2}^{-1}\right)$ configurations which is observed in all $N=125$ isotones. The effective $M 1$ operator as specified in Ref. [18] yields a good description of all known single-particle $g$ factors in the model space except for the $v p_{1 / 2}$ orbital, where it badly fails. A modification of the $v p_{1 / 2} M 1$ matrix element normalized to the $g$ factor for the $1 / 2^{-}$in ${ }^{207} \mathrm{~Pb}$ yields excellent agreement for all $N=125$ isotones including ${ }^{214} \mathrm{Ac}$ (see open red square in Fig. 10, upper panel). It should be noted that, for the odd-odd actinium isotopes, the calculated energy of the levels with spin and parity $(4-5)^{+}$in ${ }^{214} \mathrm{Ac}$ and $(4-7)^{+}$in ${ }^{212} \mathrm{Ac}$ are within $200 \mathrm{keV}$. While the spin assignment $5^{+}$for the ground state of ${ }^{214} \mathrm{Ac}$ coincides with the experimental value, for ${ }^{212} \mathrm{Ac}$ the $4^{+}$state is predicted to be the lowest in energy. However, for the comparison with the $g$ factor the $I=7^{+}$nuclear-spin state was used.

A comparison of the $g$ factor and the $Q$ values for the $N=126$ isotones is shown in Fig. 11. The $g$-factor value for astatine $(Z=83)$ was included to compare the general trend of the isotones and was taken from the high-spin isomeric state with nuclear spin $I=(21 / 2)^{-}$with known $g$ factor $g=$ $0.910(9)$. The theoretical results agree with the experimental data and suggest the extension of the closed-shell nature of the 


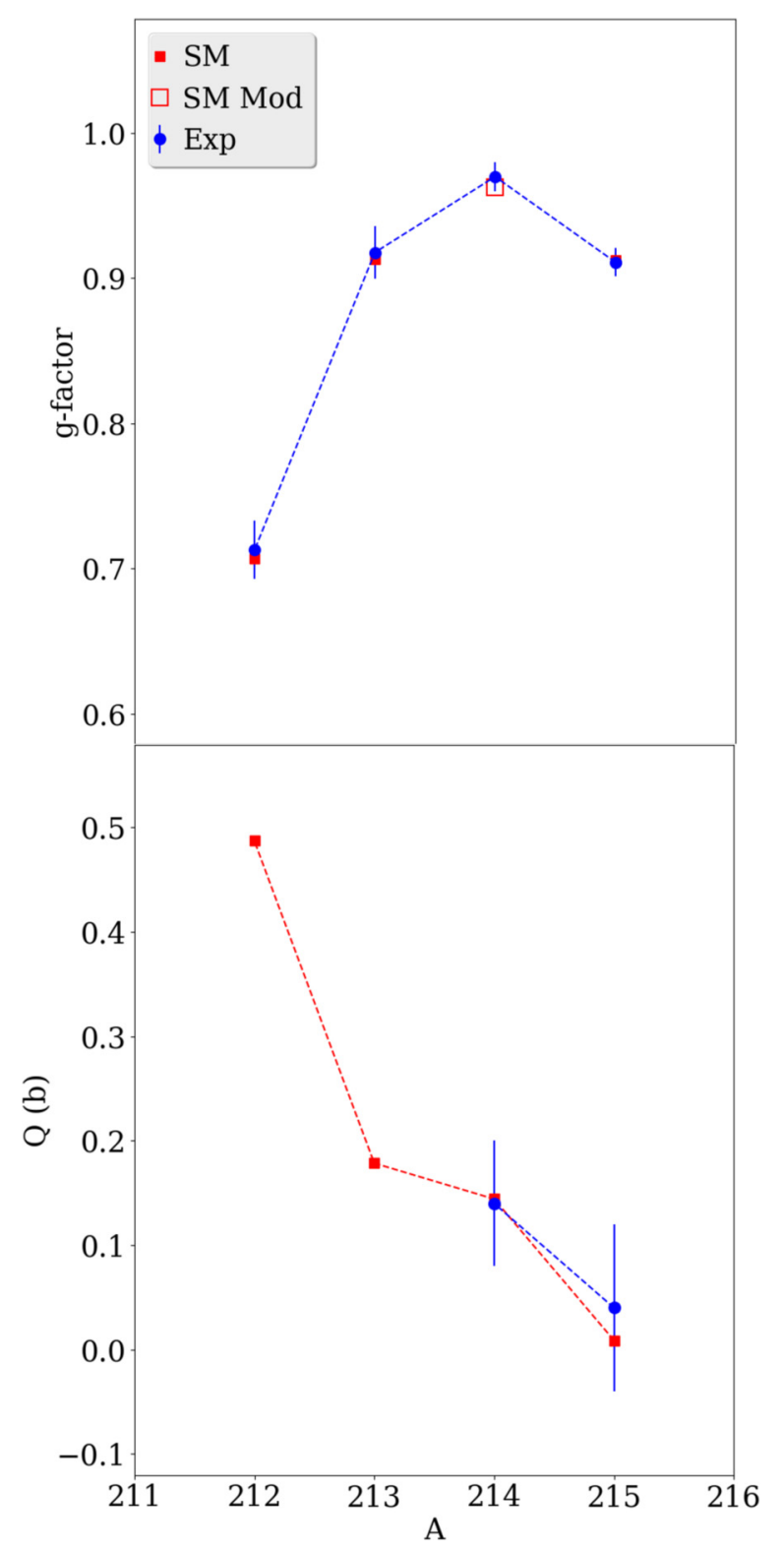

FIG. 10. Comparison between experimental $g$ factors and electric-quadrupole moments with the respective values extracted from large-scale nuclear-shell-model calculations (SM) and the modified SM (SM Mod) for the isotopes of actinium studied in this work. Here only the statistical errors are shown.

$N=126$ shell up to ${ }^{215} \mathrm{Ac}$. The linear behavior of $Q$ and nearly constant values of the $g$ factors and thus of $\mu$ is connected with the dominant $\pi h_{9 / 2}$ single-proton configuration. In terms of the independent-particle model, a linear trend of quadrupole moments as a function of the atomic number $Z$ is expected and with zero quadrupole moment in this approach being located at ${ }^{213} \mathrm{Fr}$, which corresponds to the half filled shell. The nearly zero quadrupole moment for the isotone ${ }^{215} \mathrm{Ac}$ arises from the delay in the filling of the $\pi h_{9 / 2}$ orbital due to the occupation of the $\pi f_{7 / 2}$ and $\pi i_{13 / 2}$ subshells [1]. A similar behavior for the electric-quadrupole moment for the $N=125$ isotones is shown in the lower panel of Fig. 11. The linear trend for the

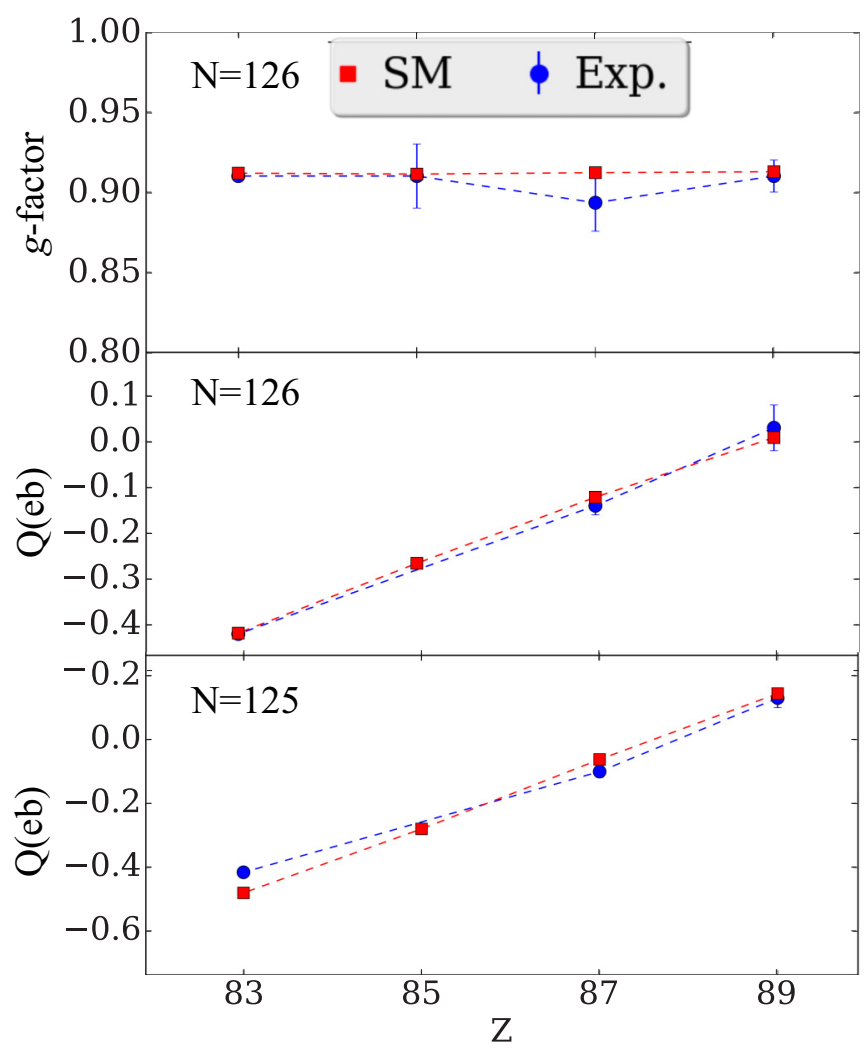

FIG. 11. A comparison between experimental $g$ factors and electric-quadrupole moments with the respective values extracted from large-scale nuclear shell-model calculations $N=126$ closedshell isotones. In the lower panel the comparison between the experimental quadrupole moments with the respective values extracted from shell-model calculations for the $N=125$ isotones is shown. The value $Q=-0.415$ (17) b for ${ }^{209} \mathrm{Bi}$ was taken from Ref. [54] and was used to correct the electric-quadrupole moment of ${ }^{208} \mathrm{Bi}$ based on the correction for the previous value from ${ }^{209} \mathrm{Bi}$ [55]. In the same way as in the case of $h_{9 / 2}\left({ }^{215} \mathrm{Ac}\right)$, the $g$ factor for the ground state in ${ }^{211} \mathrm{At}$ was taken from $g$ factors of the high-spin isomeric state $I=(21 / 2)^{-}$ [9]. Only the statistical errors are shown.

$N=125$ isotones reflects the dominant character of the single proton in the $h_{9 / 2}$ subshell with respect to the quadrupole moment as the contribution from neutron $p_{1 / 2}$ orbital is zero.

\section{CONCLUSION}

The hyperfine structure of ${ }^{212-215} \mathrm{Ac}$ was measured by using the in-gas-cell laser ionization spectroscopy method and studies on ${ }^{214,215} \mathrm{Ac}$ were carried out for the first time under on-line conditions by using the in-gas-jet laser ionization spectroscopy method. Direct comparison of both experimental methods showed a superior performance of the in-gas-jet method in terms of resolution, efficiency, and selectivity. Under optimized experimental conditions for the spatial and temporal overlapping of the laser light with the gas jet the total efficiency for the in-gas-jet method can reach more than $10 \%$. Hyperfine coupling constants were extracted for ${ }^{212-215} \mathrm{Ac}$ and $g$ factors 
and $Q$ values were deduced. Data from this work and from previous works on francium, astatine, and bismuth isotopes with the same neutron number were compared with large-scale nuclear shell-model calculations. The linear trend in the $Q$ values for the $N=126$ and $N=125$ isotones as a function of proton number indicates that the nuclear structure of these isotopes is determined by the unpaired proton in the $\pi h_{9 / 2}$ shell and suggests a closed-shell nature at $N=126$ up to ${ }^{215} \mathrm{Ac}$. Large-scale nuclear shell-model calculations using ${ }^{208} \mathrm{~Pb}$ as a core are able to reproduce the $g$ factors and $Q$ values for all the isotopes reported in this paper. Further investigation on the $N \simeq 126$ isotopes of thorium and protactinium will be performed by using the S3-Low-Energy Branch at the Grand Accelerateur National d'Ions Lourds (GANIL) [23].

\section{ACKNOWLEDGMENTS}

This work was supported by FWO-Vlaanderen (Belgium), by GOA/2010/010 (BOF KU Leuven), by the IAP Belgian Science Policy (BriX network P7/12), and by a Grant from the European Research Council (ERC-2011-AdG-291561HELIOS). S.S. acknowledges a Ph.D. Grant from the Belgian Agency for Innovation by Science and Technology (IWT). L.P.G. acknowledges FWO-Vlaanderen (Belgium) as an FWO Pegasus Marie Curie Fellow. S.F. and R.B. acknowledge the support by the German Ministry for Education and Research (BMBF) under Contract No. 05P15SJCIA. This work was supported by the European Commission within the European Nuclear Science and Application Research ENSAR2 (Grant Agreement No. 654002).
[1] E. Caurier, M. Rejmund, and H. Grawe, Phys. Rev. C 67, 054310 (2003).

[2] K. Heyde and J. L. Wood, Rev. Mod. Phys. 83, 1467 (2011).

[3] J. Khuyagbaatar, A. Yakushev, C. E. Düllmann, D. Ackermann, L.-L. Anderson, M. Block, H. Brand, D. M. Cox, J. Even, U. Forsberg et al., Phys. Rev. Lett. 115, 242502 (2015).

[4] P. Campbell, I. D. Moore, and M. R. Pearson, Prog. Part. Nucl. Phys. 86, 127 (2016).

[5] T. E. Cocolios, A. N. Andreyev, B. Bastin, N. Bree, J. Büscher, J. Elseviers, J. Gentens, M. Huyse, Y. Kudryavtsev, D. Pauwels et al., Phys. Rev. Lett. 103, 102501 (2009).

[6] R. Ferrer, N. Bree, T. Cocolios, I. Darby, W. D. H. De Witte, J. Diriken, J. Elseviers, S. Franchoo, M. Huyse, N. Kesteloot et al., Phys. Lett. B 728, 191 (2014).

[7] M. Laatiaoui, W. Lauth, H. Backe, M. Block, D. Ackermann, B. Cheal, P. Chhetri, C. E. Düllmann, P. van Duppen, J. Even et al., Nature (London) 538, 495 (2016).

[8] Y. Kudryavtsev, R. Ferrer, M. Huyse, P. V. den Bergh, and P. V. Duppen, Nucl. Instrum. Methods Phys. Res., Sect. B 297, 7 (2013).

[9] National Nuclear Data Center Database ENSDF: http://www.nndc.bnl.gov.

[10] D. J. Decman, H. Grawe, H. Kluge, and K. H. Maier, Z. Phys. A: At. Nucl. 310, 55 (1983).

[11] D. Decman, H. Grawe, H. Kluge, K. Maier, N. R. A. Maj, Y. Agarwal, K. Blume, M. Guttormsen, H. Hbel, and J. Recht, Nucl. Phys. A 436, 311 (1985).

[12] W. F. Meggers, M. Fred, and F. F. Tomkins, J. Res. Natl. Bur. Stand. 58, 2763 (1957).

[13] F. S. Tomkins, M. Fred, and W. F. Meggers, Phys. Rev. 84, 168 (1951).

[14] M. Fred, F. S. Tomkins, and W. F. Meggers, Phys. Rev. 98, 1514 (1955).

[15] F. Hessberger, S. Hofmann, D. Ackermann, V. Ninov, M. Leino, S. Saro, A. Andreyev, A. Lavrentev, A. Popeko, and A. V. Yeremin, Eur. Phys. J. A 8, 521 (2000).

[16] A. Waldek, N. Erdmann, C. Grüning, G. Huber, P. Kunz, J. V. Kratz, J. Lassen, G. Passler, and N. Trautmann, AIP Conf. Proc. 584, 219 (2001).

[17] J. Rossnagel, S. Raeder, A. Hakimi, R. Ferrer, N. Trautmann, and K. Wendt, Phys. Rev. A 85, 012525 (2012).
[18] R. Ferrer, A. Barzakh, B. Bastin, R. Beerwerth, M. Block, P. Creemers, H. Grawe, R. de Groote, P. Delahaye, X. Fléchard et al., Nat. Commun. 8, 14520 (2017).

[19] Y. Kudryavtsev, M. Facina, M. Huyse, J. Gentens, P. V. den Bergh, and P. V. Duppen, Nucl. Instrum. Methods Phys. Res., Sect. B 204, 336 (2003).

[20] Y. Kudryavtsev, T. Cocolios, J. Gentens, M. Huyse, O. Ivanov, D. Pauwels, T. Sonoda, P. V. den Bergh, and P. V. Duppen, Nucl. Instrum. Methods Phys. Res., Sect. B 267, 2908 (2009).

[21] M. Facina, B. Bruyneel, S. Dean, J. Gentens, M. Huyse, Y. Kudryavtsev, P. V. den Bergh, and P. V. Duppen, Nucl. Instrum. Methods Phys. Res., Sect. B 226, 401 (2004).

[22] A. Andreyev, D. Bogdanov, V. Chepigin, A. Kabachenko, O. Malyshev, Y. Muzychka, B. Pustylnik, G. Ter-Akopian, and A. Yeremin, Nucl. Phys. A 568, 323 (1994).

[23] R. Ferrer, B. Bastin, D. Boilley, P. Creemers, P. Delahaye, E. Liénard, X. Fléchard, S. Franchoo, L. Ghys, M. Huyse et al., Nucl. Instrum. Methods Phys. Res., Sect. B 317, 570 (2013).

[24] P. V. den Bergh, S. Franchoo, J. Gentens, M. Huyse, Y. Kudryavtsev, A. Piechaczek, R. Raabe, I. Reusen, P. V. Duppen, L. Vermeeren et al., Nucl. Instrum. Methods Phys. Res., Sect. B 126, 194 (1997).

[25] S. Raeder, B. Bastin, M. Block, P. Creemers, P. Delahaye, R. Ferrer, X. Fléchard, S. Franchoo, L. Ghys, L. P. Gaffney et al., Nucl. Instrum. Methods Phys. Res., Sect. B 376, 382 (2016).

[26] A. N. Andreyev, J. Elseviers, M. Huyse, P. V. Duppen, S. Antalic, A. Barzakh, N. Bree, T. E. Cocolios, V. F. Comas, J. Diriken et al., Phys. Rev. Lett. 105, 252502 (2010).

[27] J. F. Ziegler, M. D. Ziegler, and J. P. Biersack, Nucl. Instrum. Methods Phys. Res., Sect. B 268, 1818 (2010).

[28] COMSOL: https://www.comsol.com.

[29] A. Zadvornaya, Ph.D. thesis, K.U. Leuven, 2018 (unpublished).

[30] S. Raeder, M. Dombsky, H. Heggen, J. Lassen, T. Quenzel, M. Sjödin, A. Teigelhöfer, and K. Wendt, Hyperfine Interact. 216, 33 (2013).

[31] V. Sonnenschein, Ph.D. thesis, The University of Jyväskylä, 2015 (unpublished).

[32] Y. Kudryavtsev, J. Andrzejewski, N. Bijnens, S. Franchoo, J. Gentens, M. Huyse, A. Piechaczek, J. Szerypo, P. V. Duppen, P. V. D. Bergh et al., Nucl. Instrum. Methods Phys. Res., Sect. B 114, 350 (1996). 
[33] R. Ferrer, V. Sonnenschein, B. Bastin, S. Franchoo, M. Huyse, Y. Kudryavtsev, T. Kron, N. Lecesne, I. Moore, B. Osmond et al., Nucl. Instrum. Methods Phys. Res., Sect. B 291, 29 (2012).

[34] W. Gins, SATLAS: Statistical Analysis Toolbox for Laser Spectroscopy; doi:10.5281/zenodo.33291.

[35] C. Granados, Ph.D. thesis, University of Leuven, 2018 (unpublished).

[36] A. Corney, Atomic and Laser Spectroscopy (Oxford University Press, 2006)

[37] T. Sonoda, T. Cocolios, J. Gentens, M. Huyse, O. Ivanov, Y. Kudryavtsev, D. Pauwels, P. Van den Bergh, and P. Van Duppen, Nucl. Instrum. Methods Phys. Res., Sect. B 267, 2918 (2009).

[38] M. Seliverstov, T. Cocolios, W. Dexters, A. Andreyev, S. Antalic, A. Barzakh, B. Bastin, J. Büscher, I. Darby, D. Fedorov et al., Phys. Lett. B 719, 362 (2013).

[39] S. Raeder (unpublished).

[40] D. Zwarts and P. W. M. Claudemans, Z. Phys. A: At. Nucl. 320, 487 (1985).

[41] A. P. Byrne, G. D. Dracoulis, S. J. Poletti, U. Bonn, and P. Bag, Nucl. Phys. A 448, 137 (1986).

[42] P. Raghavan, At. Data Nucl. Data Tables 42, 189 (1989).

[43] I. Budinčević, J. Billowes, M. L. Bissell, T. E. Cocolios, R. P. De Groote, S. De Schepper, V. N. Fedosseev, K. T. Flanagan, S. Franchoo, R. F. Garcia Ruiz et al., Phys. Rev. C 90, 014317 (2014).
[44] A. Coc, C. Thibault, F. Touchard, H. Duong, P. Juncar, S. Liberman, J. Pinard, J. Lermé, J. Vialle, S. Bättgenbach et al., Phys. Lett. B 163, 66 (1985).

[45] J. Kilgallon, M. Pearson, J. Billowes, P. Campbell, U. Georg, I. Grant, M. Keim, R. Neugart, M. Neuroth, and S. Wilbert, Phys. Lett. B 405, 31 (1997).

[46] G. Neyens, Rep. Prog. Phys. 66, 633 (2003).

[47] F. Hessberger, S. Hofmann, I. Kojouharov, D. Ackermann, S. Antalic, P. Cagarda, B. Kindler, B. Lommel, R. Mann, A. Popeko et al., Eur. Phys. J. A 15, 335 (2002).

[48] E. Arnold, W. Borchers, M. Carre, H. T. Duong, P. Juncar, J. Lerme, S. Liberman, W. Neu, R. Neugart, E. W. Otten et al., Phys. Rev. Lett. 59, 771 (1987).

[49] E. Gomez, S. Aubin, L. A. Orozco, G. D. Sprouse, E. IskrenovaTchoukova, and M. S. Safronova, Phys. Rev. Lett. 100, 172502 (2008).

[50] L. R. C. Ekstrom and A. Rosen, Phys. Scr. 34, 624 (1986).

[51] T. Baştuğ, B. Fricke, M. Finkbeiner, and W. Johnson, Z. Phys. D: At., Mol. Clusters 37, 281 (1996).

[52] E. K. Warburton and B. A. Brown, Phys. Rev. C 43, 602 (1991).

[53] B. A. Brown, MSU-NSCL Report, OXBAXH for Windows (2004).

[54] T. Q. Teodoro and R. L. A. Haiduke, Phys. Rev. A 88, 052504 (2013).

[55] J. Bieroń and P. Pyykkö, Phys. Rev. Lett. 87, 133003 (2001). 